clinical and biological manifestations. Comparisons between different subgroups were performed with Chi-2 tests and ANOVAs.

Results We included 509 patients in the analyses, mainly women (78\%). Mean ( \pm SD) age at APS diagnosis was 36.3 \pm 14.7 years, and mean follow-up duration after APS diagnosis was $10.3 \pm 8.5$ years. Cluster hierarchical classification yield in four homogenous groups of patients. Their main characteristics are described in Table 1.

1. Cluster $1 \quad(n=181)$ included mostly patients with venous thrombosis $(78 \%)$ and premature births due to placenta insufficiency (14\%) history, without associated auto-immune disease (only $2.2 \%$ ).

2. Cluster $2(n=130)$ included older patients (mean 45.8 years), less frequently women (65\% of women), with arterial events history (89\%). Valvular involvement (21\%), migraine (21\%), livedo (35\%), arterial hypertension (49\%), and cardiovascular risk factors were relatively frequent.

3. Cluster $3(n=102)$ included younger patients, frequently women (mean 30.7 years; 92\% women), with associated SLE $(76 \%)$ or other autoimmune diseases $(30 \%)$. They frequently had history of venous thrombosis (74\%) and of pregnancy morbidity (36\%). Thrombocytopenia (48\%), haemolytic anaemia (14\%), and lupus anticoagulant (84\%) were frequent.

4. Cluster $4(n=96)$ included mainly patients with a history of CAPS (77\%) and/or APS-associated nephropathy (93\%), and pregnancy morbidity (36\%). Renal hypertension (61\%), livedo (36\%), seizures (14\%), valvular involvement (44\%) and triple positivity (66\%) were relatively frequent.

Conclusions Using an unsupervised clustering method, our study highlighted four distinct homogenous subgroups of APS patients that were predominantly venous; arterial; associated with SLE or other autoimmune disease; and microthrombotic. It confirms the underlying idea of heterogeneous pathophysiological mechanisms.

\section{O30 COMPLEMENT DEPOSITION, C4D, ON PLATELETS IS ASSOCIATED WITH VASCULAR EVENTS AND ANTIPHOSPHOLIPID ANTIBODIES IN SYSTEMIC LUPUS ERYTHEMATOSUS}

\footnotetext{
${ }^{1}$ Elisabet Svenungsson, ${ }^{1} J o h a n n a T$ Gustafsson, 'Giorgia Grosso, ${ }^{2}$ Marios Rossides, ${ }^{1}$ Iva Gunnarsson, ${ }^{3}$ Kerstin Jensen-Urstad, ${ }^{4}$ Anders Larsson, ${ }^{5}$ Kristina Nilsson-Ekdahl, ${ }^{5}$ Bo Nilsson, ${ }^{6}$ Anders A Bengtsson, ${ }^{7}$ Christian Lood. ${ }^{1}$ Division of Rheumatology, Dept. of Medicine Solna, Karolinska Institutet, Karolinska University Hospital, Stockholm, Sweden; ${ }^{2}$ Division of Clinical Epidemiology, Dept. of Medicine Solna, Karolinska Institutet, Stockholm, Sweden; ${ }^{3}$ Dept. of Clinical Physiology, Södersjukhuset, Karolinska Institutet, Stockholm, Sweden; ${ }^{4}$ Dept. of Medical Sciences, Clinical Chemistry, Uppsala University, Uppsala, Sweden; ${ }^{5}$ Dept. of Immunology, Genetics and Pathology, Uppsala University, Uppsala, Sweden; ${ }^{6}$ Dept. of Clinical Sciences Lund, Section of Rheumatology, Lund University, Lund, Sweden; ${ }^{7}$ Dept. of Medicine, Division of Rheumatology, University of Washington, Seattle, USA
}

10.1136/lupus-2020-eurolupus.41

Objective Complement components, including $\mathrm{C} 4 \mathrm{~d}$, can be found on activated platelets, a process associated with vascular disease in systemic lupus erythematosus (SLE). We investigated whether platelet $\mathrm{C} 4 \mathrm{~d}$ (PC4d) adds additional value to traditional and known lupus-associated risk factors when identifying SLE patients with vascular disease.

Method This cross-sectional study, included 308 well-characterized SLE patients and 308 matched general population controls. PC4d deposition was analyzed using flow cytometry. Values $>95 \%$ of controls were considered as PC4d positive (+). Antiphospholipid antibodies (aPL) were determined by Luminex, and the lupus anticoagulant (LA) test was performed by the DRVVT test. History of vascular disease (composite and as separate outcomes) was defined at inclusion.

Results SLE patients had increased PC4d deposition as compared to population controls $(50 \%$ versus $5 \%, \mathrm{p}<0.0001)$. $\mathrm{PC} 4 \mathrm{~d}+$ positively associated with any vascular events, and separately with venous and cerebrovascular events, and also with all investigated aPL profiles. The association for any vascular event remained statistically significant after adjustment for traditional and SLE-associated risk factors (OR:2.4, 95\% CI 1.3-4.6, p=0.006). Compared to patients negative for both PC4d and LA, patients with double positivity were more likely to have vascular disease (OR:12.0, 95\% CI 5.428.3; attributable proportion due to interaction $0.7,95 \% \mathrm{CI}$ 0.4-1.1) (figure 1).

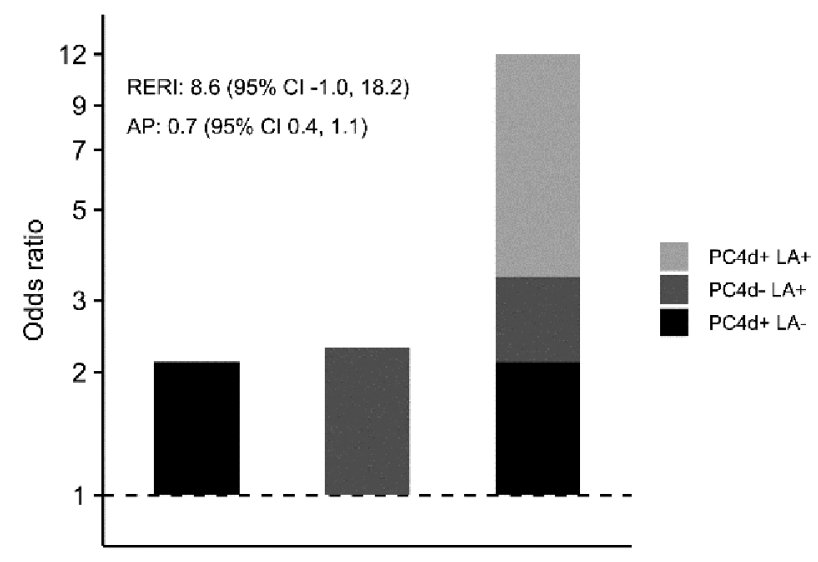

Abstract 030 Figure 1 Interaction analysis between PC4d and Lupus Anticoagulant Interaction between complement C4d deposition (PC4d) and lupus anticoagulant (LA), after adjustment for age (in 10 years), sex, hypertension, estimated glomerular filstration rate (eGFR) according to Modification of Diet in renal Disease (MDRD, per 10 units), and smoking, on the odds of vascular disease (arterial and/or venous) in individuals with systemic lupus erythematosus. RERI = relative excess risk due to interaction; $A P=$ attributable proportion due to interaction. See supplementary material for figures.

Conclusions PC4d+ is associated with vascular events in SLE, independently of traditional and SLE-associated risk factors. Concurrent presence of PC4d and LA seem to interact to further increase the odds for vascular events. Prospective studies should examine whether the aPL/PC4d combination can improve prediction of vascular events in SLE and/or APS. 